\title{
Progress of Focusing X-ray and Gamma-ray Optics for Small Animal Imaging
}

M. J. Pivovaroff, T. Funk, W. C. Barber, B. D. Ramsey, B. H. Hasegawa

August 8, 2005

Penetrating Radiation Systems and Applications VII San Diego, CA, United States August 1, 2005 through August 4, 2005 
This document was prepared as an account of work sponsored by an agency of the United States Government. Neither the United States Government nor the University of California nor any of their employees, makes any warranty, express or implied, or assumes any legal liability or responsibility for the accuracy, completeness, or usefulness of any information, apparatus, product, or process disclosed, or represents that its use would not infringe privately owned rights. Reference herein to any specific commercial product, process, or service by trade name, trademark, manufacturer, or otherwise, does not necessarily constitute or imply its endorsement, recommendation, or favoring by the United States Government or the University of California. The views and opinions of authors expressed herein do not necessarily state or reflect those of the United States Government or the University of California, and shall not be used for advertising or product endorsement purposes. 


\title{
Progress of focusing x-ray and gamma-ray optics for small animal imaging
}

\author{
M. J. Pivovaroff ${ }^{a}$, T. Funk ${ }^{b}$, W. C. Barber $^{b}$, B. D. Ramsey ${ }^{c}$, B. H. Hasegawa ${ }^{b}$ \\ ${ }^{a}$ Lawrence Livermore National Laboratory; Livermore CA, USA \\ ${ }^{b}$ University of California, San Francisco, Department of Radiology; San Francisco CA, USA \\ ${ }^{c}$ NASA Marshall Space Flight Center; Huntsville, AL, USA
}

\begin{abstract}
Significant effort is currently being devoted to the development of noninvasive imaging systems that allow in vivo assessment of biological and biomolecular interactions in mice and other small animals. Ideally, one would like to discern these functional and metabolic relationships with in vivo radionuclide imaging at spatial resolutions approaching those that can be obtained using the anatomical imaging techniques (i.e., $<100 \mu \mathrm{m}$ ), which would help to answer outstanding questions in many areas of biomedicine.

In this paper, we report progress on our effort to develop high-resolution focusing X-ray and gamma-ray optics for small-animal radionuclide imaging. The use of reflective optics, in contrast to methods that rely on absorptive collimation like single- or multiple-pinhole cameras, decouples spatial resolution from sensitivity (efficiency). Our feasibility studies have refined and applied ray-tracing routines to design focusing optics for small animal studies. We also have adopted a replication technique to manufacture the X-ray mirrors, and which in experimental studies have demonstrated a spatial resolution of $\sim 190 \mu \mathrm{m}$. We conclude that focusing optics can be designed and fabricated for gamma-ray energies, and with spatial resolutions, and field of view suitable for in vivo biological studies. While the efficiency of a single optic is limited, fabrication methods now are being developed that may make it possible to develop imaging systems with multiple optics that could collect image data over study times that would be practical for performing radionuclide studies of small animals.
\end{abstract}

Keywords: SPECT, small animal imaging, gamma-ray optics, X-ray optics

\section{INTRODUCTION}

\subsection{Motivation for Small Animal Imaging}

Animal models, including transgenic and knock-out mice, now affect research in every area of biomedical science, ranging from the neurosciences ${ }^{1}$ to the development of drug and antibody-based therapies. ${ }^{2,3}$ The increasing importance of transgenic animal technology has resulted in the development of a variety of imaging techniques, including magnetic resonance imaging (MRI) or X-ray computed tomography (CT) designed specifically for small animal imaging. Some of the most promising methods for metabolic and functional assessments, however, are best performed using radionuclide imaging techniques such as positron emission tomography (PET) and single-photon emission computed tomography (SPECT).

In these methods, a biologically-active molecule (e.g., sugar, antibody, metabolite), is labeled with a radionuclide and injected into the body. In microPET, the radiolabel emits a positron which annihilates to produces two antiparallel $511 \mathrm{keV}$ gamma-rays which are recorded by opposing elements of a detector ring surrounding the object. While microPET offers excellent sensitivity (in the range of $1-10 \%$ for small animal imaging), and reasonable spatial resolution, the uncertainty in the position of the positron annihilation and the noncolinearity of the resultant annihilation photons limits the spatial resolution achievable with PET to approximately $1 \mathrm{~mm},{ }^{4}$ and recent research has focused on building systems that approach this limit and maximize efficiency (sensitivity). ${ }^{5-7}$ In comparison, microSPECT uses a collimated detector to image gamma-rays which are emitted by the radiopharmaceutical. While SPECT does not suffer the fundamental limitation in spatial resolution as does PET, the use of a pinhole, converging hole, or parallel hole collimator invokes a critical trade-off in spatial resolution

Send correspondence to M. Pivovaroff, E-mail: pivovaroff1@llnl.gov 


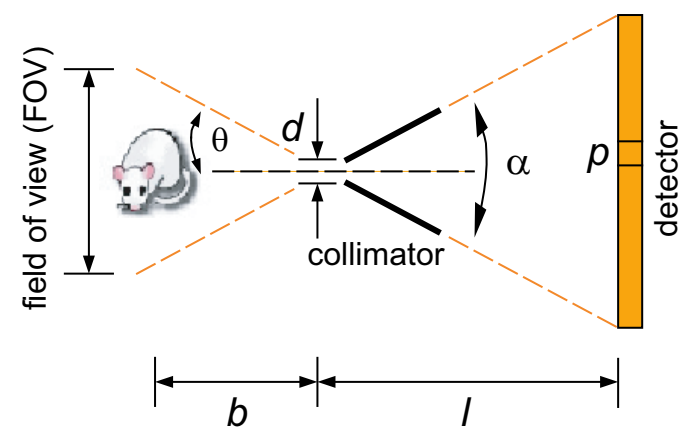

Figure 1. Basic geometry of a pinhole collimator, showing the dimensions of the pinhole aperture $d$, the field-of-view angle $\alpha$, and the separation distances between the pinhole and detector $l$ and the pinhole and object $b$.

and detection efficiency. Thus, microSPECT systems designed to offer millimeter spatial resolution are forced to operate with detection efficiencies in the range of $10^{-4}$ or poorer. The goal of this study therefore is to develop a means of performing gamma-ray imaging of small animals that would not rely on a pinhole, converging hole, or parallel hole collimator, as a method of offering the best prospect for achieving sub-millimeter spatial resolution necessary for advancing research in several fields, including cancer, cardiovascular ${ }^{8,9}$ and chronic pain ${ }^{10}$ research.

\subsection{Current Approach for SPECT}

As noted above, current methods for performing single-photon radionuclide imaging rely on absorptive collimation, with either a converging hole, parallel hole, or a pinhole collimator (shown in Figure 1) serving as the optical element. Although several groups have demonstrated spatial resolution of $1 \mathrm{~mm}$ or better with the use of single pinhole collimator, ${ }^{11-13}$ these systems have a relatively low on-axis efficiency of $g \lesssim 10^{-4}$, where

$$
g=\frac{d_{e}^{2}}{16 b^{2}} \cos ^{3} \theta
$$

and $d_{e}$ is the effective pinhole diameter, $b$ is the separation between the object and pinhole, and $\theta$ is the angle between the central axis and a position in the object. ${ }^{14}$ For photon energies below $\sim 50 \mathrm{keV}$ and systems with modest magnification, the spatial resolution $R$ is essentially equal to the effective pinhole diameter $d_{e}$. Perhaps most importantly, Equation 1, shows that $R \propto d_{e}^{2}$ and that using a smaller aperture to improve the spatial resolution unavoidably forces a profound decrease in the system efficiency.

To recoup the sensitivity lost by using a small aperture, several groups have begun developing SPECT systems that use several single-pinhole collimator-detector units ${ }^{15,16}$ or multiple parallel-hole collimator-detector units $^{17}$ in conjunction with one another or collimators with several tens of pinholes that image onto a single detector. ${ }^{18,19}$ It is still unknown whether these multipinhole methods can achieve spatial resolutions in the range of $\sim 100 \mu \mathrm{m}$ to achieve that obtained for anatomical imaging with microCT or microMRI.

\subsection{A New Approach: Focusing Optics}

Although the use of multiple pinholes appears a promising area of research, we propose a novel approach for radionuclide imaging that relies on reflective, grazing incidence mirrors instead of absorptive collimation. By using a true focusing optic, the spatial resolution achievable is decoupled from the sensitivity and depends solely on the quality of the mirrors.

In this paper, we briefly review the basic principles of X-ray optics in $\S 2$ before discussing in $\S 3$ the details of work performed during the last two years that have concentrated on improving the design tools, refining raytracing routines, and adopting a replication technique to manufacture the X-ray mirrors. In $\S 4$, we present data from our most recent prototype optics that have a measured spatial resolution of $185 \mu \mathrm{m}$, an order of magnitude better than our initial system. ${ }^{20}$ We conclude in $\S 5$ with a discussion of near-term goals and the prospects of using focusing optics for performing radionuclide studies. 


\section{BRIEF REVIEW OF REFLECTIVE X-RAY OPTICS}

\subsection{Basic Principles}

This section highlights the essential physics and concepts behind reflective, focusing X-ray and $\gamma$-ray optics. A more detailed treatment can be found elsewhere. ${ }^{20,21}$ Reflective systems rely on the total external reflection of light, a phenomenon that results from the fact that the index of refraction for all materials is less than unity at X-ray and gamma-ray energies. Typically, a high-energy photon would be simply absorbed by a slab of material. But when the light is incident at a sufficiently shallow angle, it reflects from the surface at the same grazing angle as the incident ray. This situation is analogous to the way visible light propagates down a fiber-optic cable via the total internal reflection of light. The transition between absorption and reflection occurs at a point called the critical angle, which has an inverse dependence on the photon energy and, in general, increases with the atomic number of the reflecting material. For example, the critical angle for iridium occurs at $2.0^{\circ}$ for $1 \mathrm{keV}$ $\mathrm{X}$-rays and $0.1^{\circ}$ for $100 \mathrm{keV}$ gamma-rays.

Wolter first proposed a practical optical design to take advantage of the reflective nature of materials for $\mathrm{x}$-ray and gamma-ray energies. ${ }^{22}$ He showed that pairs of mirrors built from surfaces of revolution of conic sections (e.g., hyperboloids and ellipsoids) could function together to achieve excellent focusing properties across a wide field of view (FOV). The first successful implementation of his designs was employed for an X-ray telescope in the late 1960s. ${ }^{23}$ Since then, the high-energy astrophysics community has spent billions of dollars building and refining reflective X-ray optics. An important realization of the community was that sets of cofocal mirrors could be placed inside one another, resulting in an "nested" optical system to increase the collection efficiency by orders of magnitude greater than a single pair of mirrors. For example, the European Space Agency satellite XMMNewton ${ }^{24}$ has three X-ray telescopes, each of which consists of 58 highly-nested pairs of hyperboloid-paraboloid mirrors but that functions as a single optical element.

\subsection{Practical Implementation for Radionuclide Imaging}

Although grazing incidence mirrors mainly have been utilized for telescopes (i.e., imaging at infinity), they have also been successfully used for microscopy or "near-field" applications (i.e., imaging objects located just a few meters away). ${ }^{25-27}$ Figure 2 shows the basic geometry of a Wolter optic with three nested shells intended for small animal imaging. To first order, a Wolter or grazing-incident optic is equivalent to a thin lens and has

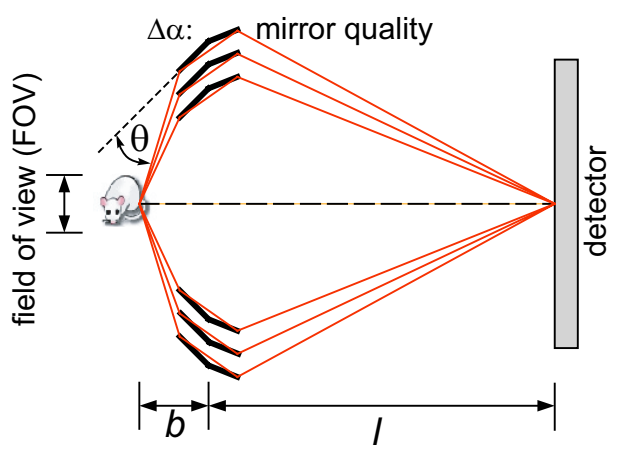

Figure 2. Schematic view of a Wolter optic with three nested mirrors, indicating the relevant parameters that determine the basic properties of the system.

similar properties. That is, the gamma-ray lens produces inverted images of the object, has a focal length $f$ given by

$$
\frac{1}{f}=\frac{1}{b}+\frac{1}{l},
$$

and a magnification $M$ defined as

$$
M \equiv \frac{l}{b}
$$


where $l$ and $b$ represent the distance between the optic and the detector and object, respectively. For an optic with $M>1$, the mirrors closest to the object will be hyperboloids, the mirrors closest to the detector will be ellipsoids. The situation is reversed if the optic has demagnification $(M<1)$.

The innermost mirror pair (those shells with the smallest radii) have the shallowest graze angles, and as more shells with larger radii are added to the optic, the graze angle also steadily increases. Because the total efficiency increases with each subsequent shell, it would be ideal to construct a lens with mirror-pairs with graze angles of many degrees. Unfortunately, once the graze angle exceeds the critical angle for a particular photon energy and material, the reflectivity of the mirrors will rapidly diminish. At this point, additional shells will not contribute to the overall efficiency of the system.

One way to extend the range of graze angles is to deposit multilayer coatings on the mirror substrates. Multilayers are alternating layers of low- and high-density materials that act like synthetic crystals, and thereby provide the substrates with reflectivity properties governed by Bragg's law. Although mulitlayers have been routinely used for normal-incidence applications for several decades, it was only in the last ten years that Christensen et al., first proposed using depth-graded multilayer structures (i.e., changing the thickness of the pairs of materials as a function of position inside the coating) for enhancing the reflectivity of grazing incidence mirrors at photon energies above $10 \mathrm{keV} .{ }^{28}$ By using well-understood multilayer combinations like Mo/Si or $\mathrm{W} / \mathrm{Si}$, it appears feasible to construct $\gamma$-ray lens systems for performing small-animal radionuclide studies by imaging the low-energy photons $(17-18 \mathrm{keV})$ emitted by agents tagged with technetium (e.g., ${ }^{95 m} \mathrm{Tc},{ }^{96} \mathrm{Tc}$, or ${ }^{99 m} \mathrm{Tc}$ ) or the $27 \mathrm{keV}$ photons emitted by ${ }^{125} \mathrm{I}$.

\section{RECENT DEVELOPMENTS}

During the last two years, we have devoted considerable resources to the development of a focusing optics specifically matched to the needs of radionuclide imaging. Two areas of research deserve particular attention: (1) a suite of design and ray-tracing tools that allows accurate prediction of system performance (e.g., efficiency and resolution) given a series of initial constraints (e.g., energy of the photons emitted by the radionuclide and separation distance between the subject and the detector) and (2) a new fabrication technique to manufacture the mirrors.

\subsection{Software Tools}

Detailed simulations are essential to formulate and test the design of reflective X-ray and $\gamma$-ray optics. Our previous work involved adopting Monte Carlo methods used for constructing X-ray telescopes ${ }^{20}$ to the specific geometry suited for small animal applications. We have built on this effort, adding significant capabilities to better predict performance and guide the choice of optical parameters for focusing optics designed for small animal imaging.

\subsubsection{Analytic Forms for Multilayer Performance}

A critical element in the design of a focusing optic is the composition and configuration of the multilayer coatings. Although multilayer reflectivity can be accurately computed from atomic physics constants and a detailed representation of the structure (e.g., the IDL-based freeware program IMD by $\mathrm{Windt}^{29}$ ), there is no easy way to determine the ideal structure parameters necessary to achieve a particular reflectance for a given photon energy and angular acceptance. In fact, it has been shown the optimal multilayer recipes are best determined from a blind, computationally-intensive search of parameter space. ${ }^{30}$

The design of the focusing optics for this study was dictated first by the range of energies that are best suited for small animal imaging with this approach (i.e., x-rays and gamma rays having energies in the range of 10-30 $\mathrm{keV}$ ), and by the choice of radioisotopes that can be used to tag existing radiopharmaceuticals (e.g., radioisotopes of technetium and iodine). We therefore have systematically studied W/Si multilayer combinations intended for focusing the $27 \mathrm{keV}$ spectral lines emitted by ${ }^{125} \mathrm{I}$ and the $17-18 \mathrm{keV}$ lines emitted by various radioisotopes of technetium. After generating reflectivity curves using the IMD package, ${ }^{29}$ a symmetric trapezoidal function, characterized by the three parameters (height, width at the base, width at the top), was fit to the data. These three parameters were then fit to third-order polynomials dependent as a function of the central graze angle, 
yielding an accurate representation of the reflectivity purely as a function of incident angle. This process provides a way to quickly and reliably calculate the total efficiency of a highly-nested system and is also crucial for speeding the Monte Carlo ray-tracing simulations needed to study the point spread function (PSF) of the lens.

\subsubsection{Performance Modelling}

We also have used Monte Carlo ray-tracing methods to study how the point spread function (PSF), and hence the spatial resolution, depends on the mirror quality and choice of optical design. To first order, these two factors are independent of one another, and can be considered separately. Imperfections in the mirror will cause the reflected photons to deviate from their intended trajectory, resulting in a broadening of the expected PSF. The performance of X-ray mirrors are usually dominated by longer spatial frequency errors (i.e., at length scales longer than $0.1 \mathrm{~mm}$ ), typically referred to as figure errors, and the actual PSF is complex and cannot be described by a simple expression. As a result, performance usually is characterized by a commonly accepted metric called the half power diameter (HPD), defined as the diameter of the smallest circle than encompasses half of the focused flux. As mirror metrology is usually characterized by the angular resolution or quality $\Delta \alpha$, the extent the mirror deviates lights, the key for predicting the spatial resolution is to relate the HPD to $\Delta \alpha$. After simulating optics spanning the range of possible configurations through Monte Carlo ray-tracing algorithms, we have determined an empirical expression for on-axis performance:

$$
\mathrm{HPD}=1.4 D \tan (\Delta \alpha) \frac{1}{1+M},
$$

where $D$ is the total separation between the object and detector

$$
D=l+b
$$

in the geometry shown in Figure 2.

Parameters in the optical design include (1) the length of the mirror, (2) the source distance $l$ and detector distance $b$ (and hence, $f$ and $D$ ) and (3) the type of design. In general, the HPD will degrade for position located increasing distances away from the central axis, with the magnitude increasing for longer mirrors. Another effect that can degrade the HPD occurs if the hyperbolic and elliptical substrates are approximated by straight lines. Although this simplification introduces aberrations that broaden the PSF, it can reduce the complexity of fabricating the optics. Use of the approximation makes sense when the mirror quality $\Delta \alpha$ is large enough that the optical aberration is small compared to the HPD expected from Equation 4, as is the case for slumped glass.

\subsection{Substrate Manufacturing}

\subsubsection{Limitations of Segmented Glass}

Initial optics were fabricated from thermally-formed glass substrates. ${ }^{20}$ The process of slumping sheets of ultrasmooth glass into segments that are then coated with multilayers and assembled into an optic is well-developed ${ }^{31}$ and has been used to achieve sub-arcminute mirror quality ${ }^{32}\left(\Delta \alpha=40^{\prime \prime}\right)$. While these substrates meet the goals of the astrophysics community and will be used in the upcoming NASA mission NuSTAR, ${ }^{33}$ they have three major drawbacks for radionuclide imaging. First, the smallest radius possible with glass is $\sim 40 \mathrm{~mm}$, but to maximize efficiency requires producing mirrors with radii $\gtrsim 10 \mathrm{~mm}$. Second, for the smallest radii substrates, great effort will be needed to achieve the angular quality better than $\sim 70^{\prime \prime}$. Inserting typical values of $D=1-4 \mathrm{~m}$ and $M=1-5$ into Equation 4 gives a HPD or spatial resolution of no better than $1 \mathrm{~mm}$. Third, glass cannot easily be shaped into the conic sections needed for a true Wolter design. Instead, the segments are cone-shaped, further degrading the resolution. For all of these reasons, a new substrate technology is desirable.

\subsubsection{Electroformed Nickel Replicated Mirrors}

A very promising approach developed by the astronomy community is the fabrication of optics via replication of individual shells from a high fidelity master mandrel. In fact, this technique was pioneered by researchers in Italy ${ }^{34-36}$ and was used to construct the telescopes for the aforementioned XMM-Newton satellite. During the last decade, NASA Marshall Space Flight Center (MSFC) has refined the process and developed significant 


\section{Mandrel Preparation}

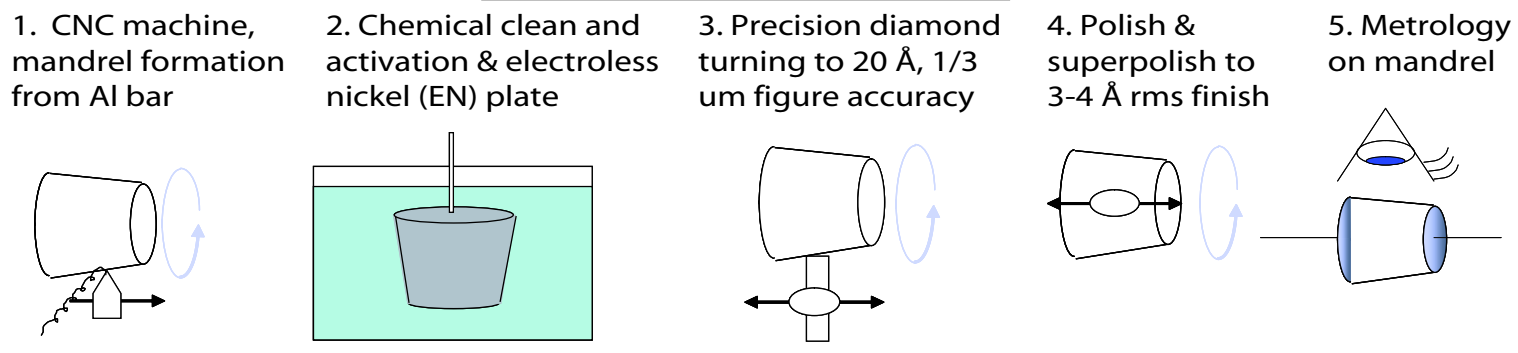

\section{Shell Fabrication}

\begin{abstract}
6. Ultrasonic clean and passivation to remove surface contaminants
\end{abstract}

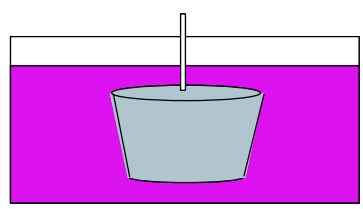

7. Deposit multilayers on mandrel

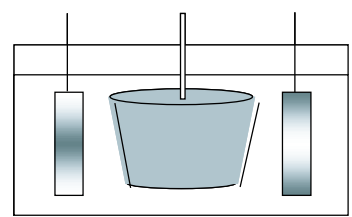

8. Electroform $\mathrm{Ni} / \mathrm{Co}$ shell onto mandrel

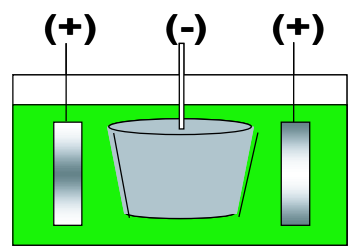

9. Separate optic from mandrel in cold water bath

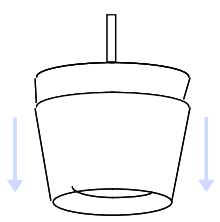

Figure 3. Diagrams indicating each stage of the manufacturing process needed to create a multilayer-coated, reflective $\gamma$-ray mirror (lens) via replication.

infrastructure and experience. ${ }^{37-40}$ Recently, we have leveraged the MSFC capabilities to manufacture prototype optics specifically intended for small-animal radionuclide imaging.

The production process involves several discrete steps that are described below and illustrated in Figure 3 . The method can broadly be divided into two main parts: mandrel preparation and shell fabrication. First, a mandrel is ground from a solid aluminum piece to a radius $\sim 0.1 \mathrm{~mm}$ smaller than that desired for the particular mirror shell (Step 1). Next, the mandrel is coated with $0.175 \mathrm{~mm}$ of electroless nickel (Step 2), a material with hardness properties suitable for precision figuring via diamond turning (Step 3) and polishing (Step 4). Finally, the mandrel is measured to verify it meets the specification of a surface finish (micro-roughness) of $\sigma=3-4 \AA$ and a figure quality of $10-15^{\prime \prime}$ (Step 5). Metrology is crucial to each step of the process and a variety of techniques and apparatus, including a long-trace profilometer, a ZEISS coordinate measuring machine, a PNEUMO and a WYKO, are used to characterized the mandrel properties.

Once the mandrel is complete, it is cleaned in a ultrasonic bath and passivated to facilitate release of the replicated shell from the mandrel (Step 6). Next, the multilayer coating is deposited on top of the mandrel and release agent (Step 7). The coated mandrel is immersed in a plating tank, and over the coarse of half a day, a $0.2 \mathrm{~mm}$ thick $\mathrm{Co} / \mathrm{Ni}$ alloy shell is formed around the mandrel (Step 8). Finally, the coated mandrel is placed in a cool water bath, and the difference between the thermal coefficient of expansion of aluminum and nickel, causes the mandrel to shrink more than the nickel mirror, freeing the optic from its master (Step 9). Great care is needed to ensure a uniform shell thickness and minimize stress imparted to the shell during the electroforming that takes place in Step 8. Any stress can lead to the deformation of the optic once it is released from the mandrel, which in turns degrades the resolution of the mirror. Significant research has gone into controlling the current density during electroplating and the resultant stress variations along a released shell is less than $\pm 3 \times 10^{5} \mathrm{~Pa}$.

\section{RESULTS FROM A REPLICATED FOCUSING LENS}

\subsection{Prototype Optic}

To prove the feasibility of using replicated optics for radionuclide imaging, a prototype lens consisting of a single shell was constructed at NASA MSFC. Although mirrors with angular quality of $\Delta \alpha=15^{\prime \prime}$ are regularly produced 
for astrophysical applications, ${ }^{40}$ an optic intended for microscopy has a much smaller radius and different shape. Since this represented the first attempt to fabricate this type of lens, the specification on quality was reduced to $\Delta \alpha \geqq 30^{\prime \prime}$. Given that the resolution predicted from this optic from Equation 4 is $\geqq 125 \mu \mathrm{m}$, it was justified to use a Wolter-approximation optical design. Table 1 lists the important optical design parameters and Figure 4 shows pictures of the mandrel and two shells replicated from it. Finally, it is important to note that since the primary objective was to demonstrate the excellent spatial resolution possible, the process skipped the deposition of multilayer coatings (step 7 in Figure 3 ) due to resource constraints. Instead, the optic was coated with a $350 \AA$ thick film of iridium to provide high-reflectivity up to $10 \mathrm{keV}$.
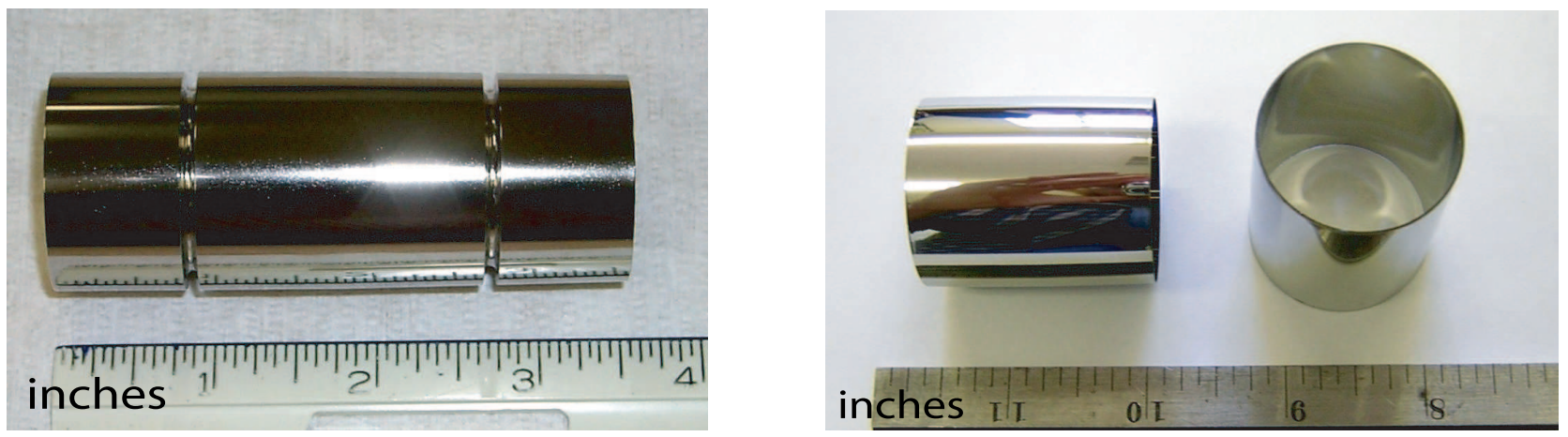

Figure 4. Left: Photograph of the mandrel after diamond turning (Step \#3 in Figure 3). The mandrel went through the remaining steps of the process except for the deposition of multilayers (Step \#7 in Figure 3). Right: Photograph of two individual optics replicated off the mandrel shown to the left. The optics were coated with $350 \AA$ of iridium to enhance reflectivity.

Table 1. Properties of the prototype replicated optic

\begin{tabular}{lr}
\hline \hline Property & Value \\
\hline Design type & Wolter approximation \\
Separation distance, $D$ & $3.0 \mathrm{~m}$ \\
Magnification, $M$ & 4.0 \\
Optic length & $42.7 \mathrm{~mm}$ \\
Central radius & $16.8 \mathrm{~mm}$ \\
Central graze angle & $0.5^{\circ}$ \\
Geometric efficiency & $4.5 \times 10^{-6}$ \\
Mirror quality, $\Delta \alpha$ & $\geqq 30^{\prime \prime}$ \\
Coating & $350 \AA$ Ir; (no multilayers) \\
\hline \hline
\end{tabular}

\subsection{Initial X-ray Tests}

\subsubsection{Experimental Apparatus}

The testing protocol used in this study followed protocols developed to evaluate lenses made earlier from thermally-formed glass ${ }^{20}$; in addition, they utilized custom fabricated structures to mount and align the optic with respect to the source and detector. Here, we quickly review the essential aspects of the experiments.

The spatial resolution was measured using a microfocus X-ray tube as the source and phosphor-coated CCD camera as the detector. The tube has a tungsten anode with an elliptical spot $100 \mu \mathrm{m}$ wide by $65 \mu \mathrm{m}$ tall and was operated at an anode potential of $40 \mathrm{kV}$. The camera consists of a $1024 \times 1024$ pixel CCD read-out device coupled by an optical taper to a $\mathrm{Gd}_{2} \mathrm{O}_{2} \mathrm{~S}$ scintillator screen. The effective pixel size is $70 \mu \mathrm{m}$ on a side, for a total active area $7 \mathrm{~cm} \times 7 \mathrm{~cm}$. 
The first step was to determine the mirror quality of the optic by making measurements with the lens intentionally de-focused. This is achieved simply by perturbing the distance $l$ between the x-ray source and the optic away from the focused conditions defined by Equation 2. The resulting images then are compared against those obtained from Monte Carlo ray-tracing studies in which mirror imperfections are modelled. The mirror imperfections are best modelled using scattering theory (e.g., the formulation of Beckmann and Spizzichino ${ }^{41}$ is well-suited for grazing incidence optics), and by comparing the observed pattern from the de-focused optic against those obtained from the Monte Carlo simulations, the scale-length and amplitude of the errors can be inferred. Figure 5 compares the actual data (left) with the simulated data (right), and the excellent agreement between the two validates this approach.
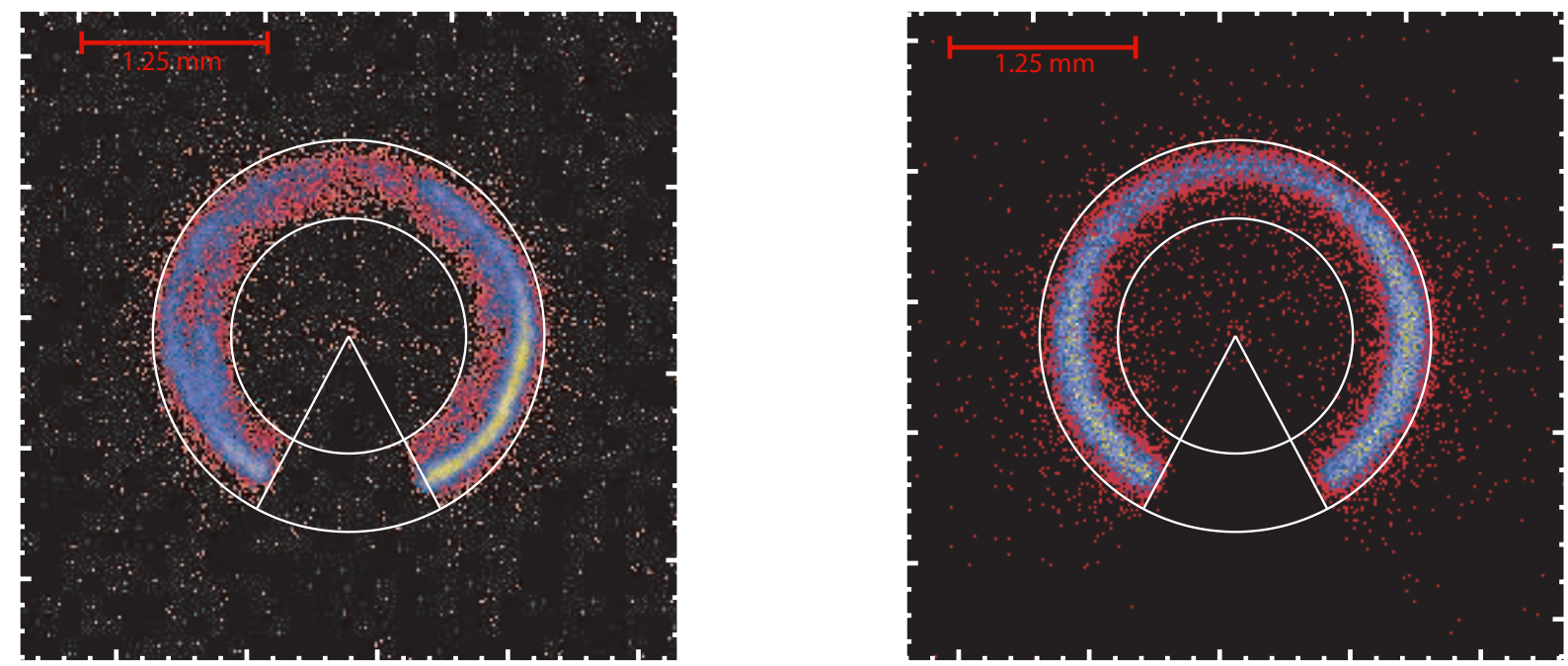

Figure 5. Left: On-axis image of the X-ray tube with detector intentionally defocused. Right: Simulated image produced from a Monte Carlo ray-tracing routine. The circles and lines are overlaid to help with the comparison. The missing "wedge" of emission results from obscuration by the support structure that is used to hold the optic.

Next, in order to map out the sensitivity distribution of the optic, the X-ray tube was rastered systematically across the FOV in $1.0 \mathrm{~mm}$ steps in the horizontal and vertical direction. Figure 6 (left) shows a false-color composite image made from the source positioned at 25 discrete locations $(\mathrm{X}=\{0, \pm 1, \pm 2 \mathrm{~mm}\}, \mathrm{Y}=\{0, \pm 1$, $\pm 2 \mathrm{~mm}\})$. Overlayed are contour plots from a simulation that rasters the $\mathrm{X}$-ray tube in exactly the same fashion. To better the see the excellent agreement between the measured data and predicted performance, Figure 6 (right) compares the false-color image of the X-ray tube on axis with the contours generated from the simulation. The majority of the flux falls in a ellipse just larger than the X-ray spot, with a small fraction scattered into an extended halo due to figure errors.

Further evidence of the validity of the ray-tracing comes from comparing the integrated intensity in each of the spots with one another. A monotonic decrease is expected in the efficiency as a source moves across the FOV. This trend occurs for two reasons. First, there are losses due to vignetting, a purely geometric phenomenon where photons do meet the conditioning for focusing. Second, there are losses due to the angular dependence on the reflectivity curve. For sources at the edge of the FOV, one of the reflections requires occurs at an incidence angle much larger than the critical angle, where the reflectivity will be much lower than unity.

To properly model the positional dependence on flux requires approximating the spectrum incident on the lens with a simple functional form. The optic has a high-energy cut-off of $\sim 10 \mathrm{keV}$ due to the iridium coating and the graze angle of $0.5^{\circ}$. The bremsstrahlung spectrum emerging from the tube travels $0.6 \mathrm{~m}$ to the optic and another $2.4 \mathrm{~m}$ after it has been focused, and the lower energy photons will largely be absorbed by the $3 \mathrm{~m}$ of air. Thus, we assumed the spectrum is Gaussian in shape, centered at $8 \mathrm{keV}$ with a $1 \mathrm{keV}$ FWHM. Table 2 compares the measured flux, normalized to the central $\mathrm{X}=0 \mathrm{~mm}, \mathrm{Y}=0 \mathrm{~mm}$ position, with those predicted by the ray-tracing simulations. The percentages to the left of a column are the measured values, the ones to the 

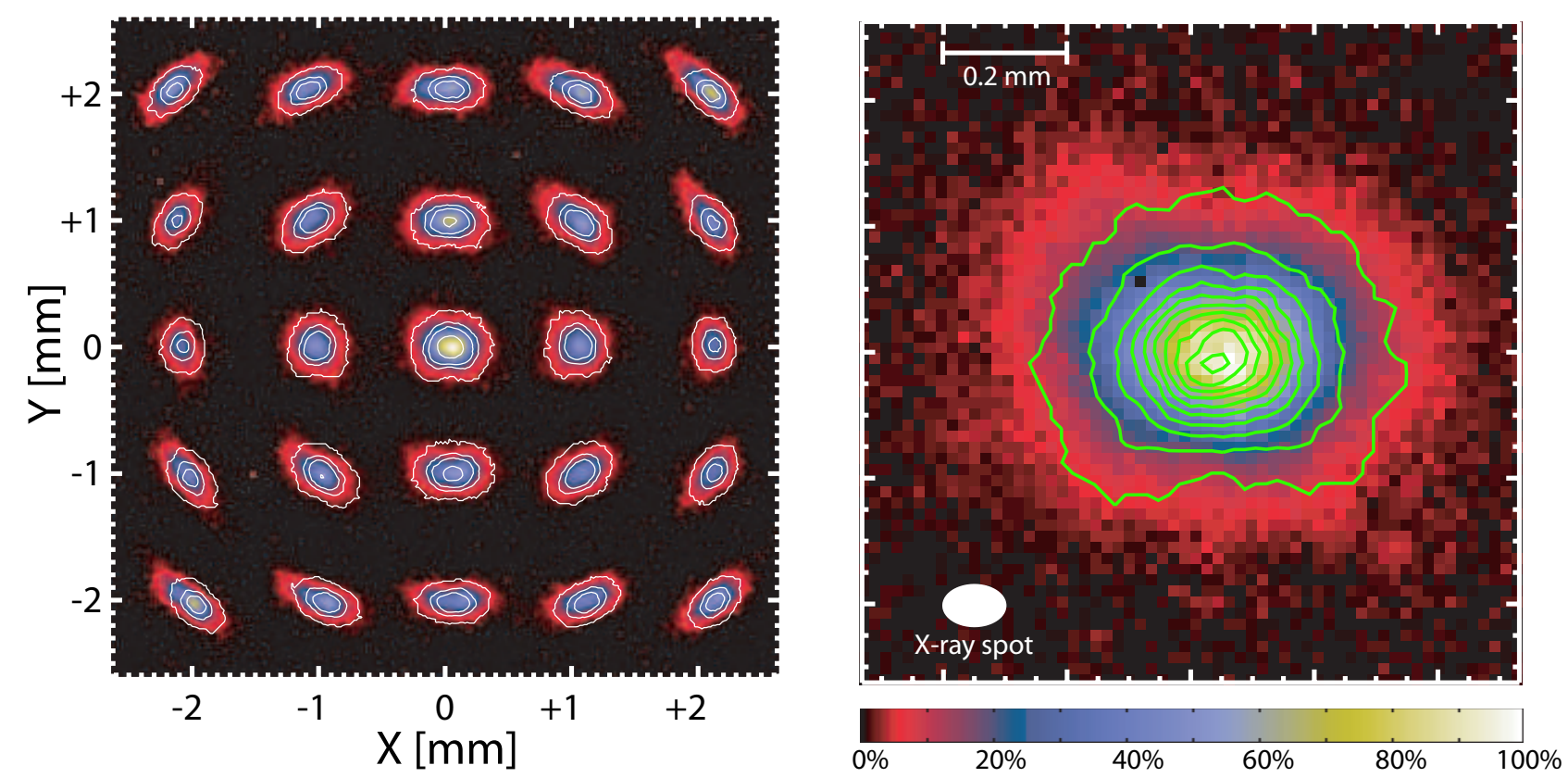

Figure 6. Left: False-color composite image made up from 25 individual exposures with the X-ray tube systematically rastered across the FOV. The contours trace the distribution prediction by the Monte Carlo simulations. Right: Falsecolor image of the X-ray source placed on-axis. The contours trace the simulated image and and are drawn in $10 \%$ steps spanning from $5 \%$ to $95 \%$ of maximum intensity. The excellent agreement indicates the scattering parameters used to characterize the optical quality of the lens are tuned properly.

right are simulated. There is uniform agreement, within the $\pm 1 \%$ statistical uncertainty of both the measured and simulated values. The slightly higher efficiency at the positions with $Y>0 \mathrm{~mm}$ results from a mounting structure that supports the optic and blocks photons. The effect of the structure is visible in Figure 5.

Table 2. Measured vs. simulated flux as a function of position

\begin{tabular}{|c|c|c|c|c|c|}
\hline & $\mathrm{X}=-2 \mathrm{~mm}$ & $\mathrm{X}=-1 \mathrm{~mm}$ & $\mathrm{X}=\mathbf{0} \mathrm{mm}$ & $\mathrm{X}=+1 \mathrm{~mm}$ & $\mathrm{X}=+2 \mathrm{~mm}$ \\
\hline $\mathbf{Y}=-2 \mathbf{m m}$ & $20 \% \quad 20 \%$ & $29 \% \quad 27 \%$ & $32 \% \quad 31 \%$ & $28 \% \quad 28 \%$ & $21 \% \quad 21 \%$ \\
\hline $\mathbf{Y}=-1 \mathbf{m m}$ & $20 \% \quad 19 \%$ & $41 \% \quad 37 \%$ & $57 \% \quad 55 \%$ & $40 \% \quad 40 \%$ & $21 \% \quad 20 \%$ \\
\hline $\mathbf{Y}=0 \mathbf{m m}$ & $18 \% \quad 20 \%$ & $49 \% \quad 45 \%$ & $100 \% \quad 100 \%$ & $44 \% \quad 48 \%$ & $16 \% \quad 20 \%$ \\
\hline $\mathbf{Y}=+1 \mathbf{m m}$ & $23 \% \quad 23 \%$ & $46 \% \quad 44 \%$ & $70 \% \quad 69 \%$ & $41 \% \quad 46 \%$ & $17 \% \quad 23 \%$ \\
\hline $\mathbf{Y}=+2 \mathbf{m m}$ & $23 \% \quad 23 \%$ & $33 \% \quad 31 \%$ & $39 \% \quad 36 \%$ & $32 \% \quad 31 \%$ & $20 \% \quad 23 \%$ \\
\hline
\end{tabular}

Percentages on the left are measured, those on the right are simulated

\subsection{Derived Resolution}

The resolution of the optic can be evaluated two ways. First, we measure the extent of the X-ray spot when it imaged on-axis by the lens. Figure 7 (left) again shows the measured spot, now overlayed with a circle the size of the HPD and a half-power ellipse: the smallest ellipse that has the same aspect ratio as the spot that contains $50 \%$ of the focused photons. The computed dimensions are similar: the HPD is $315 \mu \mathrm{m}$, while the ellipse is $370 \mu \mathrm{m} \times 240 \mu \mathrm{m}$, a factor of $3.7 \times$ larger than the spot.

Because the source used to calibrate the lens is extended, the spatial resolution of the mirrors is better than the $\sim 300 \mu \mathrm{m}$ size of the focused spot. To better understand the true performance of the optic, we performed ray-trace simulations of a point source moved across the FOV using the scattering parameters determined from the de-focused data. Figure 7 (right) shows the HPD as a function of off-axis position. The spatial resolution 

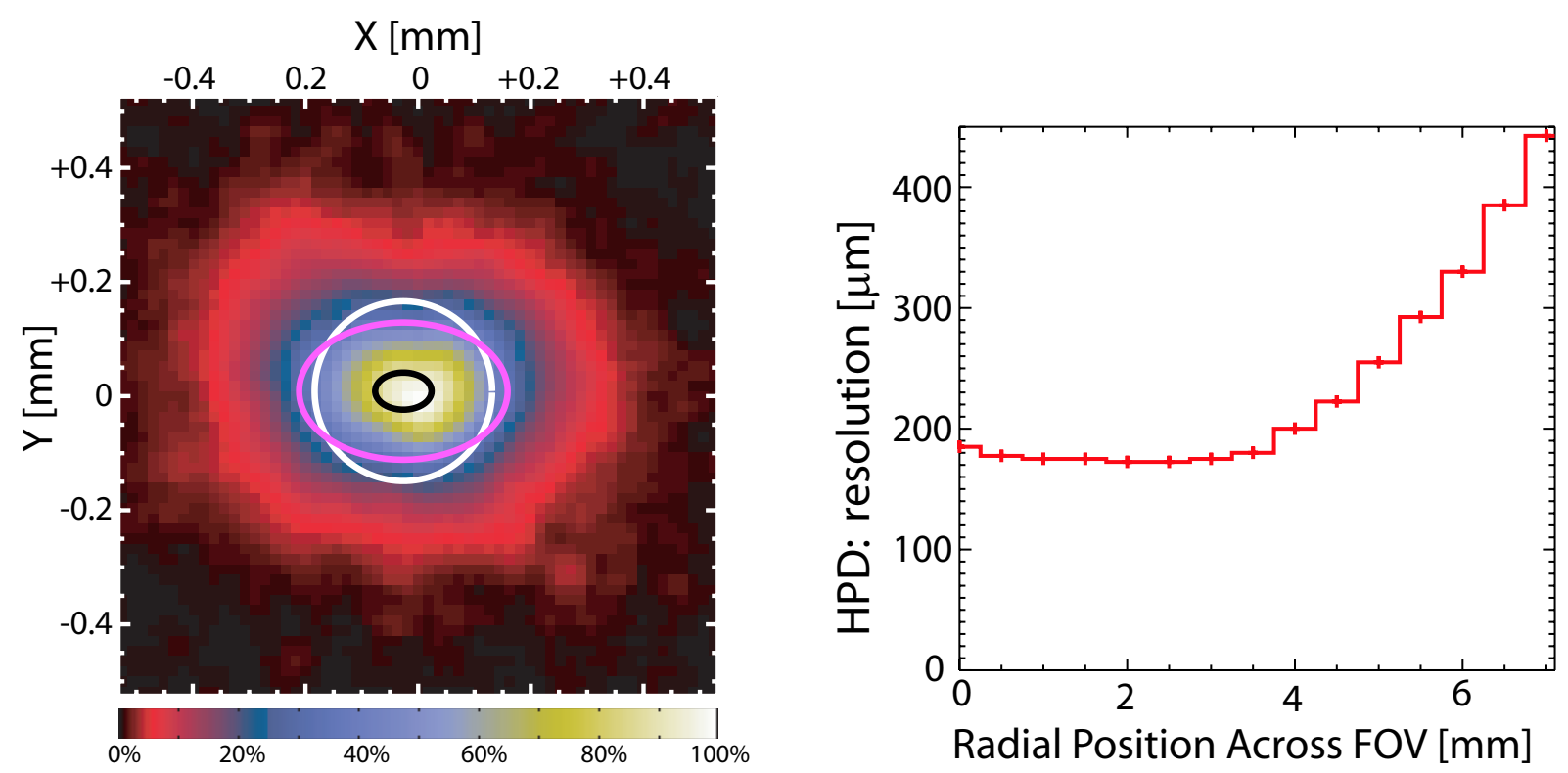

Figure 7. Left: Smoothed $(3 \times 3$ pixel $)$ on-axis image of the X-ray tube spot. The small ellipse shows the size of the actual spot. The larger ellipse and circle represents the region that captures $50 \%$ of the flux. Right: Simulated resolution (HPD) as a function of radial position across the FOV. The resolution is $185 \mu \mathrm{m}$ across the central $1 \mathrm{~cm}$ region, and slowly degrades from the aberration inherent to the Wolter approximation optical design.

has a constant value of $185 \mu \mathrm{m}$ across the central $1 \mathrm{~cm}$ portion of the FOV and gradually degrades to $400 \mu \mathrm{m}$ at the edge of the $1.4 \mathrm{~cm} \mathrm{FOV}$.

To explore the imaging capabilities, we performed a simulation with four, identical compact sources, $100 \mu \mathrm{m}$ in diameter located, at position located at position of $\mathrm{Y}=0 \mathrm{~mm}$, and $\mathrm{X}=\{0 \pm 0.15 \mathrm{~mm}\}$ and $\mathrm{X}=-3.0 \pm 0.15$. Figure 8 shows the false-color image of the four sources (left) and the compression of the data in the vertical direction (right). It is clear that the optic can easily distinguish objects $100 \mu \mathrm{m}$ in size that are separated by just $300 \mu \mathrm{m}$.

\section{FUTURE PROSPECTS AND CONCLUSION}

\subsection{Near-term Goals}

These results prove that it possible to achieve spatial resolution as good as $200 \mu \mathrm{m}$ with a focusing gamma-ray lens. The next step is to fabricate an optic with the same process, but to an include a multilayer coating (Step 7, Figure 3) engineered to focus $27 \mathrm{keV}$ photons. This will allow imaging of a phantom or other source (e.g., a BrachySeed ${ }^{\mathrm{TM}}$ ) that contains an agent tagged with ${ }^{125} \mathrm{I}$ to demonstrate the feasibility of using reflective optics for radionuclide imaging.

A complete lens with efficiency of $10^{-5}-10^{-4}$ is possible with a full complement of $40-80$ nested mirrors. The exact sensitivity will depend on the radionuclide to be studied (the multilayers for the 17 or $18 \mathrm{keV}$ lines emitted by Tc have better reflectivity than those engineered for focusing the higher energy lines from ${ }^{125} \mathrm{I}$ ), and the final spatial resolution and FOV will be tuned for the specific biological study to be performed.

In developing these designs, we considered the number of shells needed to obtain sufficiently high efficiency, as well as the cost of manufacturing the optic. During the optic fabrication, each shell requires a unique mandrel that has a current price of approximately $\$ 10,000$ USD. The total cost, then, of the first complete lens could approach $\$ 500,000$ USD. There are, fortunately, three mitigating circumstances that will drive down the expense for future optics. First, during the initial development, figuring and polishing techniques will be optimized for the small mirrors required for radionuclide imaging, lowering the price of each mandrel. Second, once a mandrel is produced, many tens of shells can be replicated from it, with minimal polishing and cleaning needed between 

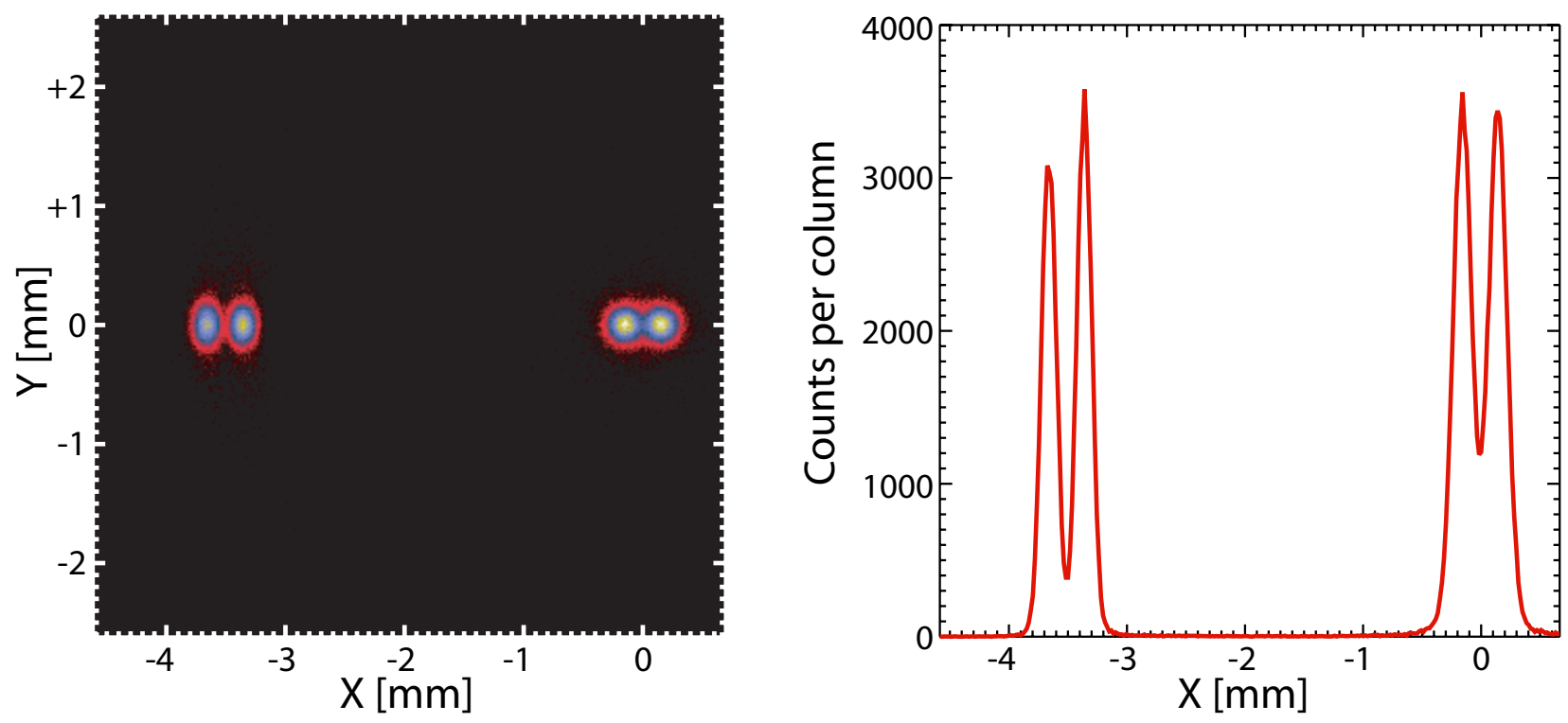

Figure 8. Left: Simulation of four equal-strength $100 \mu \mathrm{m}$ diameter sources. Right: Simulated data summed in the vertical direction. Although the sources are only located $300 \mu \mathrm{m}$ apart, they are easily resolved by the optic.

replications. This makes the first optic quite expensive, and subsequent ones more and more affordable. Third, we have just engineered a promising, inexpensive approach for making high-quality substrates from plastic. This process was developed for X-ray optics intended for a particle astrophysics experiment at CERN (the CERN Axion Solar Telescope $[\mathrm{CAST}]^{42}$ ) but could be applicable to biomedical applications as well.

\subsection{Prospects}

Looking ahead, much effort will be required in optimizing the way data are acquired and how images are reconstructed. One benefit of using reflective optics is that, unlike with absorptive collimation techniques, data sets should be free of scattered events. This situation is a natural result of using multilayer coatings, as the mirrors will only properly focus photons that satisfy both a particular angular and energy requirement. This inherent property of scatter rejection therefore should reduce this unwanted background signal in the images, improve signal contrast, and thereby make it easier to detect weaker sources.

It is also clear that the PSF of the optic should be incorporated into the reconstruction techniques. The benefit of this additional calibration has been recognized by several groups, and characterization of the PSF is now standard practice for many of the latest SPECT systems. ${ }^{16,19}$ It will be particularly important to perform this step when using focusing optics due to noticeable change in response across the FOV. Although the algorithms will necessarily be different than those used already in use for SPECT or other tomographic modalities, it should be possible to adopt techniques already in use to analyze images obtained from reflective $\mathrm{X}$-ray telescopes. However, compensating the data for the geometric characteristics of the PSF should produce images that have reduced noise and are more uniform in their resolution characteristics than those that are obtained by simple reconstruction of raw data produced by the optic.

Finally, focusing gamma-ray optics likely can achieve spatial resolutions that are better than the values of 100-200 $\mu \mathrm{m}$ targeted in this study. We expect that this could occur as substrate manufacturing technology continues to improve in a way that allows mirror quality improve to $\Delta \alpha=2-10^{\prime \prime}$. For systems with a limited FOV (e.g., 5-10 mm in diameter), it would be possible to achieve spatial resolution of $10-20 \mu \mathrm{m}$ with an efficiency of $10^{-5}-10^{-4}$. While such a system might eventually be feasible technically, it still is uncertain whether it would be practical for biological applications where the images necessarily must be produced with a minimal level of contrast and statistical quality. In turn, these charateristics are determined by the relative and absolute radiopharmaceutical distributions in the target and background regions, and by the collection efficiency of the optic. If the gamma-ray lens has a fixed efficiency, then, at some point the number of photons per 
voxel would require an activity concentration that would perturb the genetic, immunological, or other biological process that is being studied, or that at high radiopharmaceutical concentrations might approach a lethal dose to the organism.

However, this currently is also a concern with conventional forms of radionuclide imaging such as microSPECT or microPET. Moreover, if the imaging study hopes only to detect the presence of an agent, localized to a particular region, the requirements are different. As recently shown by Cherry, ${ }^{43}$ the detection limits obtained for a microPET system vary significantly depending on if the radionuclide-tagged object is point-like (much smaller than the system spatial resolution) or is extended (much larger than the system resolution). Such work indicates that a ultra-high-resolution SPECT system based on true focusing optics could be useful when the goal is find small numbers of cells in an organ, a capability that could find application in many research areas.

\section{ACKNOWLEDGMENTS}

This work was supported in part by grant KP1401030, issued by the U.S. Department of Energy (DoE), and by Contract No. W-7405-ENG-48, issued by the U.S. DoE, Office of Biological and Environmental Research, by the University of California, Lawrence Livermore National Laboratory. The project also received generous support from the University of California, Office of the President, under the auspices of the Campus-Laboratory Collaboration (CLC) program. The project has used resources supported in part by NIH grant 5 R01 EB00348, "Imaging Structure and Function in Small Animals."

\section{REFERENCES}

1. X. Gao, A. Kemper, and B. Popko, "Advanced transgenic and gene-targeting approaches," Neurochem Res 24, pp. 1181-1188, 1999.

2. J. A. Gossen, W. J. de Leeuw, and J. Vijg, "LacZ transgenic mouse models: their application in genetic toxicology," Mutation Res 307, pp. 451-459, 1994.

3. D. Hanahan, "Transgenic mice as probes into complex systems," Science 246, pp. 1265-1275, 1989.

4. F. J. Beekman, A. P. Colijn, B. Vastenhouw, V. M. Wiegant, and M. A. F. M. Gerrits, "High-resolution emission tomography of small laboratory animals: physics and gamma-astronomy meet molecular biology," Nucl Inst Meth A 509, pp. 229-234, 2003.

5. C. Knoess et al., "Performance evaluation of the microPET R4 PET scanner for rodents," Eur J Nucl Med Mol Imaging 30, pp. 737-747, 2003.

6. Y. C. Tai et al., "MicroPETII: design, development and initial performance of an improved microPET scanner for small-animal imaging," Phys Med Biol 48, pp. 1519-1537, 2003.

7. J. Missimer, Z. Madi, C. Keller, A. Schubiger, and S. M. Ametamey, "Performance evaluation of the 16module quad-HIDAC small animal PET camera," Phys Med Biol 49, pp. 2069-2081, 2004.

8. M. W. Dae, J. W. O'Connell, E. H. Botvinick, and M. C. Chin, "Acute and chronic effects of transient myocardial ischemia on sympathetic nerve activity, density, and norepinephrine content," Cardiovasc Res $\mathbf{3 0}$, pp. 270-280, 1995.

9. N. M. Caplice and B. J. Gersh, "Stem cells to reapir the heart: a clinical perspective," Circ Res 92, pp. 6-8, 2003.

10. J. G. Burke, R. W. Watson, D. McCormack, F. E. Dowling, M. G. Walsh, and J. M. Fitzpatrick, "Intervertebral discs which cause low back pain secrete high levels of proinflammatory mediators," J Bone Joint Surg Br 84, pp. 196-201, 2002.

11. M. C. Wu, B. H. Hasegawa, and M. W. Dae, "Peformance evaluation of a pinhole SPECT system for myocardial perfusion imaging of mice," Med Phys 29, pp. 2830-2839, 2002.

12. F. J. Beekman, D. P. McElroy, F. Berger, S. S. Gambhir, E. J. Hoffman, and S. R. Cherry, "Toward in vivo nuclear microscoopy: iodine-125 imaging in mice using micro-pinholes," Eur J Nucl Med 29, pp. 933-938, 2002.

13. D. P. McElroy et al., "Performance evaluation of A-SPECT: a high resolution desktop pinhole SPECT system for imaging small animals," IEEE Trans Nucl Sci 49, pp. 2139-2147, 2002. 
14. J. A. Sorenson and M. E. Phelps, Physics in Nuclear Medicine, W. B. Saunders Company, Philadelphia, 1987.

15. S. D. Metzler, R. J. Jaszczak, N. H. Patil, S. Vemulapalli, G. Akabani, and B. B. Chin, "Molecular imaging of small animals with a triple-head SPECT system using pinhole collimation," IEEE Trans Med Imaging 24, pp. 853-862, 2005.

16. L. R. Furenlid, D. W. Wilson, Y. C. Chen, H. Kim, P. J. Pietraski, M. J. Crawford, and H. H. Barrett, "FastSPECTII: A second-generation high-resolution dynamic SPECT imager," IEEE Trans Nucl Sci 51, pp. 631-635, 2004.

17. W. S. Choong, W. W. Moses, C. S. Tindall, and P. N. Luke, "Design for a high-resolution smallanimalSPECT system using pixellated $\mathrm{Si}(\mathrm{Li})$ detectors for in vivo ${ }^{125} \mathrm{I}$ imaging," IEEE Trans Nucl Sci $\mathbf{5 2}$, pp. 174-180, 2005.

18. N. U. Schramm, G. Ebel, U. Engeland, T. Schurrat, M. Béhé, and T. M. Behr, "High-resolution SPECT using multipinhole collimation," IEEE Trans Nucl Sci 50, pp. 315-320, 2004.

19. F. J. Beekman, F. van der Have, B. Vastenhouw, A. J. A. van der Linden, P. P. van Rijk, J. P. H. Burbach, and M. P. Smidt, "U-SPECT-I: A novel system for submillimeter-resolution tomography with radiolabeled molecules in mice," J Nucl Med 46, pp. 1194-1200, 2005.

20. M. J. Pivovaroff et al., "Small-animal radionuclide imaging with focusing gamma-ray optics," Proc SPIE 5199, pp. 147-161, 2004.

21. M. Pivovaroff, W. Barber, T. Funk, B. Hasegawa, C. Taylor, W. Craig, and K. Ziock, "High resolution radionuclide imaging using focusing gamma-ray optics," in Small-Animal SPECT Imaging, Plenum US, 2005.

22. H. Wolter, "Spiegelsysteme streifenden Einfalls als abbildende Optiken für Röntgenstrahlen," Ann Physik 10, pp. $94-114,1952$.

23. R. Giacconi, N. F. Harmon, R. F. Lacey, and Z. Szilagyi, "Aplanatic telescope for soft x rays," J Opt Soc Amer 55, pp. 345-347, 1965.

24. F. D. Jansen et al., "XMM-Newton observatory. I. the spacecraft and operations," Astron Astrophys 365, pp. L1-L6, 2001.

25. R. J. Ellis, J. E. Trebes, D. W. Phillion, J. D. Kilkenny, S. G. Glendinning, J. D. Wiedwald, and R. A. Levesque, "Four-frame gated Wolter x-ray microscope," Rev Sci Instr 61, pp. 2759-2761, 1990.

26. T. Onuki, K. Sugisaki, and S. Aoki, "Fabrication of Wolter type I mirror for soft x-ray," Proc SPIE 1720, pp. 258-263, 1992.

27. P. Troussel, P. Munsch, and J.-J. Ferme, "Microfocusing between 1 and $5 \mathrm{keV}$ with Wolter type optics," Proc SPIE 3773, pp. 60-69, 1999.

28. F. E. Christensen, A. Hornstrup, N. J. Westergaard, H. W. Schnopper, J. Wood, and K. Parker, "A graded d-spacing multilayer telescope for high energy x-ray astronomy," Proc SPIE 1546, pp. 160-167, 1992.

29. D. L. Windt, "IMD: Software for modeling the optical properties of multilayer films," Computers Phys 12, pp. 360-370, 1998.

30. P. H. Mao, F. A. Harrison, D. L. Windt, and F. E. Christensen, "Optimization of graded multilayer designs for astronomical x-ray telescopes," App Opt 38, pp. 4766-4775, 1999.

31. W. W. Craig, C. J. Hailey, M. Jimenez-Garate, D. L. Windt, F. A. Harrison, P. H. Mao, F. E. Christensen, and A. M. Hussain, "Development of thermally formed glass optics for astronomical hard x-ray telescopes," Opt Exp 7, pp. 178-185, 2000.

32. J. E. Koglin et al., "Hard x-ray optics: from HEFT to NuSTAR," Proc SPIE 856-867, 2004.

33. http://www.caltech. nustar. edu

34. O. Citterio et al., "Optics for the x-ray imaging concentrators aboard the x-ray astronomy satellite SAX," App Opt 27, pp. 1470-1475, 1988.

35. O. Citterio et al., "Imaging characteristics of the development model of the JET-X x-ray telescope," Proc SPIE 1546, pp. 150-159, 1991.

36. O. Citterio et al., "Results of x-ray measurements on electroformed mirror shelss for the XMM project," Proc SPIE 1742, pp. 256-263, 1993. 
37. B. D. Ramsey, D. Engelhaupt, C. O. Speegle, S. L. O’Dell, R. A. Austin, J. J. Kolodziejczak, and M. C. Weisskopf, "The HERO program, high-energy replicated optics for a hard x-ray balloon payload," Proc SPIE 3765, pp. 816-821, 1999.

38. B. D. Ramsey et al., "HERO: High-energy replicated optics for a hard x-ray balloon payload," Proc SPIE 4138, pp. 147-153, 2000.

39. B. D. Ramsey et al., "HERO: program status and first images from a balloon-borne focusing hard x-ray telescope," Proc SPIE 4496, pp. 140-145, 2002.

40. B. D. Ramsey et al., "The development of hard x-ray optics at MSFC," Proc SPIE 5168, pp. 129-135, 2004.

41. P. Beckmann and A. Spizzichino, The Scattering of Electromagnetic Waves from Rough Surfaces, Artech House Publishers, 1987.

42. K. Zioutas et al., "First results from the CERN axion solar telescope (CAST)," Phys Rev Let 94, p. 121301, 2005.

43. S. R. Cherry, "High resolution gamma ray detectors for in vivo imaging." LLNL seminar; http://rdc.1lnl.gov/seminars/Abstracts/2005_052405_cherry.html, May 2005. 